\author{
Military Technical College \\ Kobry El-Kobbah, \\ Cairo, Egypt.
}

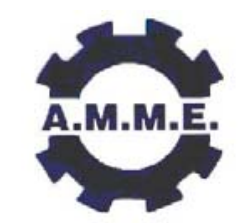

$13^{\text {th }}$ International Conference on Applied Mechanics and Mechanical Engineering.

\title{
EFFECT OF VARIABLE THERMAL CONDUCTIVITY ON HEAT TRANSFER FROM SPINES OF UNIFORM CROSS SECTIONS USING HPM
}

\author{
WAQAR A. KHAN*, AMIR SHAHZAD**
}

\begin{abstract}
Homotopy perturbation method (HPM) is employed to investigate the effects of temperature dependent thermal conductivity on the dimensionless temperature distribution along spines of arbitrary uniform cross sections (rectangular, square, elliptical and circular) in still ambient air. Thermal performance of each cross section is compared in terms of efficiency based on equal cross sectional area of the spines. It is noticed that elliptical and rectangular cross sections with smaller axis ratios have higher efficiencies and they approach to circular and square cross sections in the limiting case when their axis ratios tend to unity. The effect of Biot number on dimensionless temperature distribution and on efficiency of each cross section is also presented graphically.
\end{abstract}

\section{KEY WORDS}

Spines, Homotopy Perturbation Method, Thermal Conductivity, Spine Efficiency

* Associate Professor, Department of Mechanical Engineering, National University of Sciences and Technology, PNS Jauhar, Karachi, Pakistan. ** Research Associate, Department of Mathematics, COMSATS Institute of Information Technology, Abbottabad, Pakistan. 


\section{NOMENCLATURE}

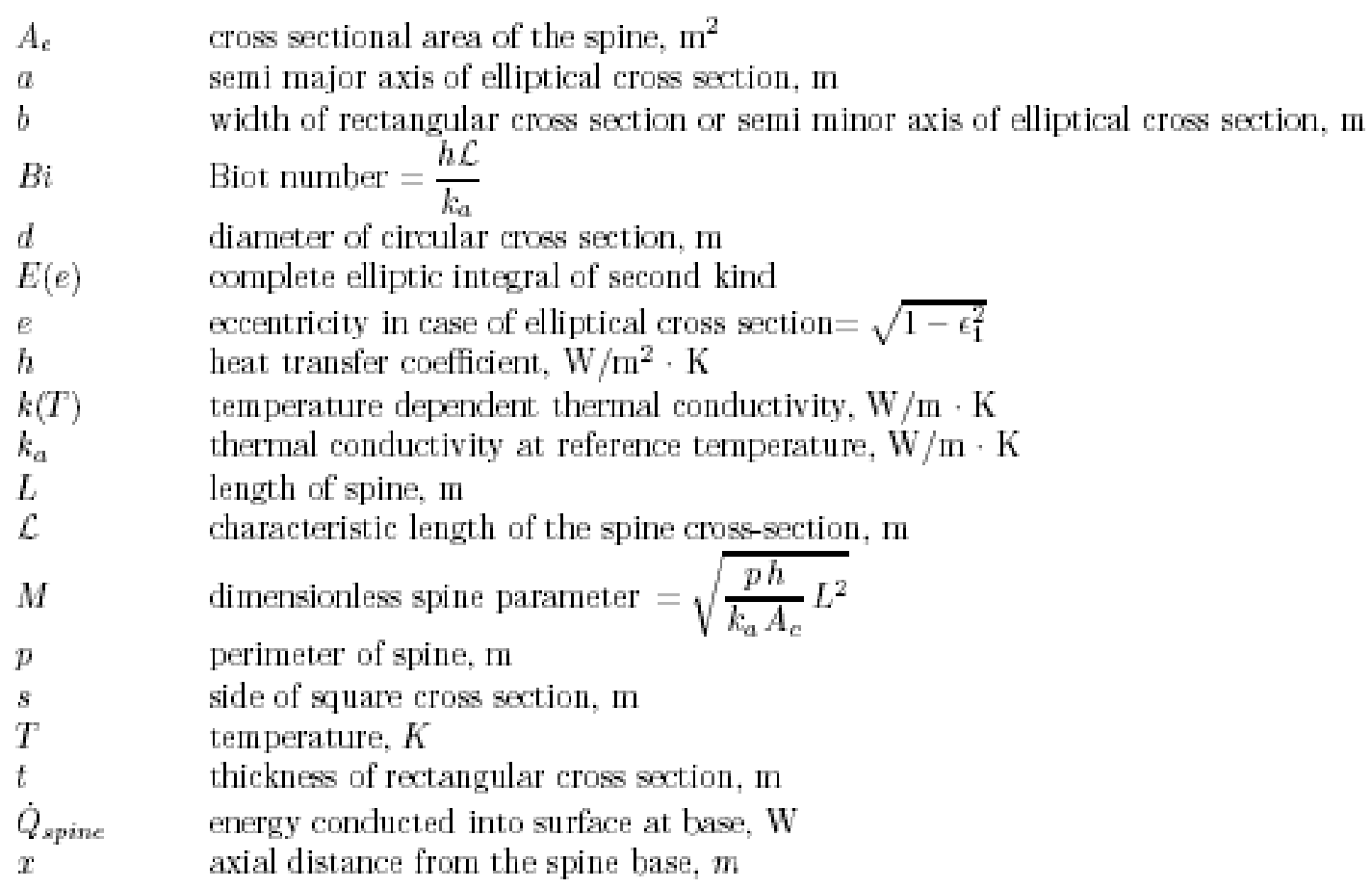

Greek Symbols

$\beta \quad$ temperature coefficient

$\Delta T \quad$ temperature difference between base and ambient, $\mathrm{K}$

$\epsilon \quad$ dimensionless temperature coefficient

$\epsilon_{1} \quad$ axis ratio for elliptical cross section $=\frac{b}{a}$

$\epsilon_{2} \quad$ axis ratio for rectangular cross section $=\frac{t}{b}$

$\eta_{\text {spine }} \quad$ spine efficiency

$\theta_{0} \quad$ initial guess

$\theta \quad$ dimensionless temperature $=\frac{T-T_{a}}{T_{b}-T_{a}}$

$\zeta \quad$ dimensionless axial distance

Subscripts

1 first order solution

2 second order solution

a ambient

$b \quad$ base

C circular cross section

E elliptical cross section

$R \quad$ rectangular cross section

$S \quad$ square cross section

$w \quad$ wall

\section{INTRODUCTION}

Heat transfer from a prime surface can be significantly increased by the addition of spines which are attached to the prime surface. These spines are widely used in several engineering applications. Different spine geometries can be found in standard heat transfer text books [1-3]. In this paper HPM is employed to investigate the effects of temperature dependent thermal conductivity on heat transfer from spines of arbitrary constant 
cross sections in still ambient air for mixed boundary conditions. Homotopy perturbation method (HPM) was first proposed by $\mathrm{He}[4]$ and has been used to solve non-linear equations in heat transfer applications by several authors including Khan and coworkers [5-7].

Zubair et al. [8] studied optimization of circular fins with general power - law profile and temperature dependent thermal conductivity. Chiu and Chen [9] irvestigated the results for a rectangular fin with variable thermal conductivity using the Adomian decomposition method. He [10, 11] introduoed homotopy perturbation method (HPM) whereas Lio [12,13] employed homotopy analysis method (HAM) to solve nonlinear equations.

Razelos and Imre [14] investigated the effect of variable thermal conductivity and heat transfer coefficient along the trapezoidal profile of an annular fin. They assumed that heat transfer coefficient increases according to a power law with distance from the fin base. Unal [15] developed the closed-form solution for the straight fin having a rectangular profile for the one-dimensional temperature distribution, assuming that the heat transfer coefficient is a power function of the difference between the temperature of the fin and the ambient fluid. Sen and Trinh [16] obtained an exact solution for the performance of the same fin and the heat transfer coefficient given by a power-type temperature dependence. Laor and Kalman [17] presented a theoreticalnumerical analysis of longitudinal and annular fins and spines having rectangular, triangular, and parabolic profiles. They analyzed the effect of a temperature-dependent heat transfer coefficient on the fin efficiency and optimum fin dimensions. The heat transfer coefficient used in this analysis was approximated by a power function of the fin and fluid temperature differenoe.

Assumptions

This study is based on the following assumptions:

1. The heat flow and temperature distribution are steady state.

2. The spine material is homogeneous and isotropic.

3. The heat transfer coefficient is constant and uniform over the entire surface of the spine.

4. The temperature of the air surrounding the spine is uniform.

5. The temperature at the base is uniform and constant.

6. There are no distributed heat souroes within the spine.

7. The radiation heat transfer is negligible.

8. Heat transfer is one-dimensional.

9. Thermal contact at the spine base is perfect.

\section{ANALYSIS}

Consider a spine of arbitrary uniform cross section (Fig. 1) with cross-sectional area $A_{c}$, perimeter $p$, length $L$, and temperature dependent thermal conductivity $k(T)$. The area and perimeter for each cross section are given in Table 1 . The spine is attached to a prime surface at constant temperature $T_{b}$ and extends into a fluid at ambient temperature $T_{a}$. Heat is removed from the surface and the tip via natural convection only. The dimension of each cross section is determined on the basis of equal cross sectional area. The Biot number is defined as $B i=\frac{h \mathcal{L}}{k_{a}}$, where $\mathcal{L}$ is the characteristic length of the spine cross-section. This characteristic length of the spine is defined as the ratio of the cross sectional area to the perimeter, $A_{c} / p$, and for different cross sections is presented in Table 1 . The Biot number is assumed to be sufficiently small that the temperature across the cross-section is essentially constant.

Steady state heat balance equation for the spine of arbitrary uniform cross section can be written as

$$
\frac{d}{d x}\left[k(T) \frac{d T}{d x}\right]=\frac{p h}{A_{c}}\left(T-T_{a}\right)
$$

with

$$
k(T)=k_{a}\left[1+\beta\left(T-T_{a}\right)\right]
$$


where $h$ is the uniform convective heat transfer coefficient, $k_{a}$ is the thermal conductivity at ambient temperature, and the constant $\beta$ is called the temperature coefficient of thermal conductivity.

In dimensionless form, Eq. (1) can be written as

$$
\frac{d}{d \zeta}\left[(1+\epsilon \theta) \frac{d \theta}{d \zeta}\right]=M^{2} \theta
$$

where $M$ is the dimensionless spine parameter and it is written as

$$
M=\sqrt{\frac{p h}{k_{a} A_{c}} L^{2}}
$$

and the dimensionless variables are

$$
\theta=\frac{T-T_{a}}{T_{b}-T_{a}}, \quad \zeta=\frac{x}{L}, \quad \epsilon=\beta \Delta T
$$

Equation (3) can be separated into linear and non-linear parts

$$
\left[\frac{d^{2} \theta}{d \zeta^{2}}-M^{2} \theta\right]+\epsilon \frac{d}{d \zeta}\left(\theta \frac{d \theta}{d \zeta}\right)=0
$$

This equation can be solved using homotopy perturbation method for the following mixed boundary conditions:

$$
\theta(0)=1 \quad \text { and } \quad \frac{d \theta(1)}{d \zeta}=-B i \theta(1)
$$

Homotopy for Eq. (6) satisfies the equation

$$
(1-q)\left[\left(\frac{d^{2} \theta}{d \zeta^{2}}-M^{2} \theta\right)-\left(\frac{d^{2} \theta_{0}}{d \zeta^{2}}-M^{2} \theta_{0}\right)\right]+q\left[\Theta \frac{d^{2} \theta}{d \zeta^{2}}+\epsilon\left(\frac{d \theta}{d \zeta}\right)^{2}\right]=0
$$

where $q \in[0,1]$ is an embedding parameter, and $\theta_{0}$ is the initial guess that can be obtained by solving the linear part of Eq. (6)

$$
\frac{d^{2} \theta_{0}}{d \zeta^{2}}-M^{2} \theta_{0}=0
$$

with boundary conditions

$$
\theta_{0}(0)=1 \quad \text { and } \quad \frac{d \theta_{0}(1)}{d \zeta}=-B i \theta_{0}(1)
$$

The solution of Eq. (9) is given by

$$
\theta_{0}=C_{1} e^{-M \zeta}+C_{2} e^{M \zeta}
$$

where $C_{1}$ and $C_{2}$ are the constants of integration and can be determined by using boundary conditions (10).

$$
\left.\begin{array}{l}
C_{1}=\frac{e^{-M}(M-B i)}{(M+B i) e^{M}+e^{-M}(M-B i)} \\
C_{2}=\frac{(M+B i) e^{M}}{(M+B i) e^{M}+e^{-M}(M-B i)}
\end{array}\right\}
$$

Assuming that Eq. (8) has a solution of the form

$$
\theta(\zeta, q)=\theta_{0}+q \theta_{1}+q^{2} \theta_{2}+\cdots
$$

By substituting Eq. (13) in Eq. (8) and equating the powers of $q$, we obtain

$$
\frac{d^{2} \theta_{1}}{d \zeta^{2}}-M^{2} \theta_{1}+\epsilon\left[\theta_{0} \frac{d^{2} \theta_{0}}{d \zeta^{2}}+\left(\frac{d \theta_{0}}{d \zeta}\right)^{2}\right]=0
$$


The solution of Eq. (14) can be written as

$$
\begin{aligned}
\theta_{1}(\zeta)= & e^{M \zeta} C_{4}+e^{-M \zeta} C_{3}-\frac{2}{3} \epsilon e^{-2 M \zeta} \\
& \left\{\frac{e^{-2 M}(M-B i)^{2} e^{4 M \zeta}}{\left[(M+B i) e^{M}+(M-B i) e^{-M}\right]^{2}}+\frac{e^{2 M}(M+B i)^{2}}{\left[(M+B i) e^{M}+(M-B i) e^{-M}\right]^{2}}\right\}
\end{aligned}
$$

Using boundary conditions

$$
\theta_{1}(0)=0 \quad \text { and } \quad \frac{d \theta_{1}(1)}{d \zeta}=-B i \theta_{1}(1)
$$

the constants $C_{3}$ and $C_{4}$ can be written as

$$
\begin{aligned}
C_{3}= & \frac{1}{3\left[(M-B i) e^{-M}+(M+B i) e^{M}\right]^{3}}\left[\left(6 M^{3}+6 B i^{3}-6 M B i^{2}-6 M^{2} B i\right) e^{-M}+\left(2 \epsilon M^{2} B i\right.\right. \\
& \left.-2 \epsilon M B i^{2}+2 \epsilon M^{3}-2 B i^{3} \epsilon+3 M^{3}-3 B i^{3}+3 M^{2} B i-3 M B i^{2}\right) e^{M}+\left(2 \epsilon M^{3}-2 B i^{3} \epsilon-\right. \\
& \left.\left.9 M^{2} B i+9 M B i^{2}+3 M^{3}-3 B i^{3}-6 \epsilon M^{2} B i+6 \epsilon M B i^{2}\right) e^{-3 M}-12 \epsilon M^{2} B i+4 B i^{3} \epsilon\right]
\end{aligned}
$$

and

$$
\begin{aligned}
C_{4}= & \frac{1}{3\left[(M-B i) e^{-M}+(M+B i) e^{M}\right]^{3}}\left[\left(-6 B i^{3}+6 M^{2} B i-6 M B i^{2}+6 M^{3}\right) e^{M}+\left(2 \epsilon M^{3}-\right.\right. \\
& \left.2 \epsilon M^{2} B i+3 B i^{3}-3 M^{2} B i-3 M B i^{2}-2 \epsilon M B i^{2}+2 B i^{3} \epsilon+3 M^{3}\right) e^{-M}+\left(9 M^{2} B i+9 M B i^{2}\right. \\
& \left.\left.+6 \epsilon M^{2} B i+6 \epsilon M B i^{2}+3 B i^{3}+3 M^{3}+2 \epsilon M^{3}+2 B i^{3} \epsilon\right) e^{3 M}-4 B i^{3} \epsilon+12 \epsilon M^{2} B i\right]
\end{aligned}
$$

Similarly, by equating the coefficients of $q^{2}$ in Eq. (8), we can write the second order problem as follows:

$$
\frac{d^{2} \theta_{2}}{d \zeta^{2}}-M^{2} \theta_{2}+\epsilon \theta_{0} \frac{d^{2} \theta_{1}}{d \zeta^{2}}+\theta_{1} \frac{d^{2} \theta_{0}}{d \zeta^{2}}+2 \epsilon\left(\frac{d \theta_{1}}{d \zeta}\right)\left(\frac{d \theta_{0}}{d \zeta}\right)=0
$$

The solution of Eq. (19) with boundary conditions

$$
\theta_{2}(0)=0 \quad \text { and } \quad \frac{d \theta_{2}(1)}{d \zeta}=-B i \theta_{2}(1)
$$

is given by

$$
\begin{aligned}
\theta_{2}(\zeta)= & C_{5} e^{M \zeta}+C_{6} e^{-M \zeta}-\frac{4}{3}\left[-\frac{1}{4}\left(-\frac{1}{2}+M \zeta\right) C_{2}^{2} C_{1} \epsilon e^{4 M \zeta}+\frac{1}{4} C_{2} C_{1}^{2}\left(M \zeta+\frac{1}{2}\right) \epsilon e^{2 M \zeta}\right. \\
& \left.+C_{4} C_{1} e^{M \zeta}-\frac{9}{16} \epsilon C_{2}{ }^{3} e^{6 M \zeta}-\frac{9}{16} \epsilon C_{1}^{3}+C_{3} C_{2} e^{5 M \zeta}\right] e^{-3 M \zeta} \epsilon
\end{aligned}
$$

where the constants $C_{1}, C_{2}, C_{3}, C_{4}$ are given by Eqs. $(12,17$, and 18$)$ and $C_{5}$ and $C_{6}$ are given by

$$
\begin{aligned}
C_{5}= & -\frac{-1}{12\left[(M-B i) e^{-M}+(M+B i) e^{M}\right]}\left[\left(12 B i+2 B i C_{2} C_{1}{ }^{2} \epsilon^{2}-12 M-9 B i \epsilon^{2} C_{1}{ }^{3}+9 M \epsilon^{2} C_{1}{ }^{3}-\right.\right. \\
& 9 B i \epsilon^{2} C_{2}{ }^{3}+9 M \epsilon^{2} C_{2}{ }^{3}+16 B i \epsilon C_{4} C_{1}-16 M \epsilon C_{4} C_{1}+16 B i \epsilon C_{3} C_{2}-16 M \epsilon C_{3} C_{2}-2 M C_{2} C_{1}{ }^{2} \epsilon^{2} \\
& \left.+2 B i C_{2}{ }^{2} C_{1} \epsilon^{2}-2 M C_{2}{ }^{2} C_{1} \epsilon^{2}\right) e^{-M}+\left(32 M \epsilon C_{4} C_{1}-16 B i \epsilon C_{4} C_{1}\right) e^{-2 M}+\left(4 \epsilon^{2} M^{2} C_{2}{ }^{2} C_{1}+\right. \\
& \left.2 M C_{2}{ }^{2} C_{1} \epsilon^{2}+4 B i \epsilon^{2} M C_{2}{ }^{2} C_{1}-2 B i C_{2}{ }^{2} C_{1} \epsilon^{2}\right) e^{M}+\left(-4 B i \epsilon^{2} C_{2} C_{1}{ }^{2} M-2 M C_{2} C_{1}{ }^{2} \epsilon^{2}+\right. \\
& \left.4 \epsilon^{2} C_{2} C_{1}{ }^{2} M^{2}-2 B i C_{2} C_{1}{ }^{2} \epsilon^{2}\right) e^{-M}+\left(27 M \epsilon^{2} C_{2}{ }^{3}+9 B i \epsilon^{2} C_{2}{ }^{3}\right) e^{3 M}+\left(-32 M \epsilon C_{3} C_{2}-\right. \\
& \left.\left.16 B i \epsilon C_{3} C_{2}\right) e^{2 M}+\left(-27 M \epsilon^{2} C_{1}{ }^{3}+9 B i \epsilon^{2} C_{1}{ }^{3}\right) e^{-3 M}\right]
\end{aligned}
$$

and

$$
C_{6}=-\frac{1}{12\left[(M-B i) e^{-M}+(M+B i) e^{M}\right]}\left\{\left[\left(32 M \epsilon C_{4} C_{1}-16 B i \epsilon C_{4} C_{1}\right) e^{-3 M}-9 B i \epsilon^{2} C_{2}{ }^{3}-\right.\right.
$$




$$
\begin{aligned}
& 9 B i \epsilon^{2} C_{1}{ }^{3}+12 B i+2 M C_{2} C_{1}{ }^{2} \epsilon^{2}+2 M C_{2}{ }^{2} C_{1} \epsilon^{2}+16 B i \epsilon C_{3} C_{2}+12 M-9 M \epsilon^{2} C_{1}{ }^{3} \\
& +2 B i C_{2} C_{1} \epsilon^{2}+16 M \epsilon C_{4} C_{1}+16 B i \epsilon C_{4} C_{1}+16 M \epsilon C_{3} C_{2}-9 M \epsilon^{2} C_{2}{ }^{3}+2 \\
& \left.B i C_{2}{ }^{2} C_{1} \epsilon^{2}\right] e^{M}+\left(4 \epsilon^{2} M^{2} C_{2}{ }^{2}-C 1+2 M C_{2}{ }^{2} C_{1} \epsilon^{2}+4 B i \epsilon^{2} M C_{2}{ }^{2} C_{1}-2 B i C_{2}{ }^{2} C_{1} \epsilon^{2}\right) e^{M}+ \\
& \left(-4 B i \epsilon^{2} C_{2} C_{1}{ }^{2} M-2 M C_{2} C_{1}{ }^{2} \epsilon^{2}+4 \epsilon^{2} C_{2} C_{1}{ }^{2} M^{2}-2 B i C_{2} C_{1}{ }^{2} \epsilon^{2}\right) e^{-M}+ \\
& \left(27 M \epsilon^{2} C_{2}{ }^{3}+9 B i \epsilon^{2} C_{2}{ }^{3}\right) e^{3 M}+\left(-32 M \epsilon C_{3} C_{2}-16 B i \epsilon C_{3} C_{2}\right) e^{2 M}+ \\
& \left.\left(-27 M \epsilon^{2} C_{1}{ }^{3}+9 B i \epsilon^{2} C_{1}{ }^{3}\right) e^{-3 M}\right\}
\end{aligned}
$$

The dimensionless temperature distribution, therefore, can be written as

$$
\begin{aligned}
\theta(\zeta)= & \lim _{q \rightarrow 1}\left(\theta_{0}+q \theta_{1}+q^{2} \theta_{2}+\cdots\right) \\
= & \frac{3}{4} e^{-3 M \zeta} \epsilon^{2} C_{1}{ }^{3}+\left(C_{4}+C_{5}-\frac{1}{3} \epsilon^{2} \zeta C_{2} M C_{1}{ }^{2}+C_{1}-\frac{1}{6} \epsilon^{2} C_{1}{ }^{2} C_{2}\right) e^{-M \zeta}+ \\
& \left(C_{3}+C_{2}+\frac{1}{3} \epsilon^{2} \zeta-C 2^{2} M C_{1}+C_{6}-\frac{1}{6} \epsilon^{2} C_{2}{ }^{2} C_{1}\right) e^{M \zeta}+\left(-\frac{2}{3} \epsilon C_{1}{ }^{2}-\frac{4}{3} \epsilon C_{4} C_{1}\right) \\
& e^{-2 M \zeta}+\left(-\frac{4}{3} \epsilon C_{3} C_{2}-\frac{2}{3} \epsilon C_{2}{ }^{2}\right) e^{2 M \zeta}+\frac{3}{4} e^{3 M \zeta} \epsilon^{2} C_{2}{ }^{3}
\end{aligned}
$$

Spine Efficiency

The efficiency of the spine is defined as the ratio of the actual heat transfer rate to the ideal heat transfer rate from the spine if the entire spine were at the base temperature and is given by

$$
\eta_{f}=\frac{\dot{Q}_{\text {spine }}}{\dot{Q}_{\text {spine, } \max }}
$$

where $\dot{Q}_{\text {spine }}$ can be obtained by evaluating the energy conducted into the surface at the base and can be written as

$$
\begin{aligned}
\dot{Q}_{\text {spine }} & =-\left.k(T) A_{c} \frac{d T}{d x}\right|_{x=0} \\
& =-\left.\frac{k_{a} A_{c}}{\mathcal{L}}\left(T_{b}-T_{a}\right)(1+\epsilon \theta) \frac{d \theta}{d \zeta}\right|_{\zeta=0}
\end{aligned}
$$

and $\dot{Q}_{\text {spine,max }}$ can be obtained by evaluating the energy convected from the spine surface to the surrounding medium and can be written as

$$
\dot{Q}_{\text {spine,max }}=h\left(A_{s}+A_{c}\right)\left(T_{b}-T_{a}\right)
$$

where $A_{s}$ is the surface area of the spine. Assuming unit length of the spine, Eq. (25) can be written as

$$
\begin{aligned}
\eta_{\text {spine }}= & -\left.\frac{1}{M^{2}(1+\mathcal{L})}(1+\epsilon \theta) \frac{d \theta}{d \zeta}\right|_{\zeta=0} \\
= & -\frac{1}{144 M(1+\mathcal{L})}\left(12+12 \epsilon C_{1} e^{M}+12 \epsilon C_{2} e^{-M}+12 \epsilon C_{4} e^{M}+12 \epsilon C_{3} e^{-M}-8 \epsilon^{2} C_{1}{ }^{2} e^{2 M}\right. \\
& -8 \epsilon^{2} C_{2}{ }^{2} e^{-2 M}-4 \epsilon^{3} M C_{2}{ }^{2} C_{1} e^{-M}+4 \epsilon^{3} M C_{2} C_{1}{ }^{2} e^{M}+12 \epsilon e^{-M} C_{6}+12 \epsilon C_{5} e^{M} \\
& -2 \epsilon^{3} C_{2}{ }^{2} C_{1} e^{-M}-16 \epsilon^{2} C_{3} C_{2} e^{-2 M}-2 \epsilon^{3} C_{2} C_{1}{ }^{2} e^{M}-16 \epsilon^{2} C_{4} C_{1} e^{2 M}+9 \epsilon^{3} C_{1}{ }^{3} e^{3 M} \\
& \left.+9 \epsilon^{3} C_{2}{ }^{3} e^{-3 M}\right)\left(2 \epsilon^{2} C_{2} C_{1}{ }^{2}+12 C_{5}+12 C_{1}+12 C_{4}-2 \epsilon^{2} C_{2}{ }^{2} C_{1}-12 C_{2}-12 C_{3}-12 C_{6}\right. \\
& \left.-32 \epsilon C_{4} C_{1}-16 \epsilon C_{1}{ }^{2}+16 \epsilon C_{2}{ }^{2}+32 \epsilon C_{3} C_{2}-27 \epsilon^{2} C_{2}{ }^{3}+27 \epsilon^{2} C_{1}{ }^{3}\right)
\end{aligned}
$$

where the constants $C_{1}$ through $C_{6}$ can be determined from Eqs. (12, 17, 18, 22, and 23).

\section{RESULTS AND DISCUSSION}

The spine performano parameter $M$ for each spine is based on the performanoe parameter of circular spine 
under the same environment and same thermal conditions. These performance parameters for each spine are presented in Table 1.

The effect of temperature dependent thermal conductivity and Biot number on dimensionless temperature distribution is shown in Fig. 2. As expected, the temperature decreases along the spine in each case for every cross section. The temperature at the tip of a circular spine is highest in each case and it decreases with the increase in Biot number, whereas the temperature for the elliptical cross section is minimum. The effect of temperature dependent thermal conductivity on temperature increases along the spine. It increases with the increase in the temperature dependent parameter $\epsilon$.

The performance of each spine for different Biot numbers is compared in Fig. 3. For smaller axis ratios $\epsilon_{1}$ and $\epsilon_{2}$, the efficiencies of rectangular and elliptical cross sections are highest and approaches to square and circular cross sections with increase in axis ratios. The increase in thermal conductivity lowers the efficiency of each cross section for a fixed Biot number. It is also noticed that the efficiency of each cross section increases with the increase in Biot number. This is mainly due to the fact that for the same length and material of the spine, Biot number depends directly on the heat transfer coefficient $h$, that increases the heat transfer and hence the efficiency of each cross section.

\section{SUMMARY AND CONCLUSIONS}

Homotopy perturbation method (HPM) is successfully employed to investigate the effects of temperature dependent thermal conductivity and Biot number on the dimensionless temperature distribution and efficiency of spines of arbitrary uniform cross sections (rectangular, square, elliptical and circular) in still ambient air. For fair comparison of different cross sections, calculations are performed for equal cross sectional areas. It is oberved that

1. Temperature at the tip of each cross section increases with increase in thermal conductivity

2. Temperature at the tip decreases with the increase in Biot number

3. Efficiency of circular cross section is lowest while the efficiencies of elliptical and rectangular sections are highest for smaller axis ratios.

4. In the limiting case, when the axis ratios $\epsilon_{1}, \epsilon_{2} \rightarrow 1$, the efficiency of the rectangular cross section approaches to that of square and the efficiency of elliptical cross section approaches to that of circular.

\section{REFERENCES}

1. Kern, D. Q., and Kraus, A. D., "Extended Surface Heat Transfer," McGraw-Hill, New York, (1972).

2. Kraus, A. D., Aziz, A., and Welty, J., "Extended Surface Heat Transfer," John Wiley, New York, (2001).

3. Aziz, A., "Conduction Heat Transfer," Heat Transfer Handbook, edited by A. Bejan and A.D. Kraus, Wiley, New York, (2003).

4. He, J. H., "Homotopy Perturbation Technique," Computational Methods in Applied Mechanics Engineering, Vol. 178, pp. 257 - 262, (1999).

5. S. Noreen, and W. A. Khan, "Effect of Slip on Flow of Non-Newtonian Fluid Down Inclined Plane Using HPM," accepted for publication in Journal of Non-Newtonian Fluid Mechanics.

6. Naila K. and W. A. Khan, "Effects of Variable Thermal Conductivity on Heat Transfer from Rotating Porous Plate Using HPN", aocepted for publication in Journal of Heat Transfer.

7. Z. H. Khan and W. A. Khan, "Effect of Variable Thermal Conductivity on Heat Transfer From a Hollow Sphere with Heat Generation", submitted to Journal of Heat Transfer.

8. Zubair, S. M., Al-Garni, A. Z., and Nizami, J. S., "The Optimal Dimensions Of Circular Spines with Variable Profile and Temperature - Dependent Thermal Conductivity," International Journal of Heat and Mass transfer, Vol. 39, pp. 2067 - 2075, (1995). 
9. Ching-Huang C., Cha'o-Kuang C., "A Desomposition Method for Solving the Convective Longitudinal Spine with Variable Thermal Conductivity," International Journal of Heat and Mass transfer, Vol. 45, pp. 2067 - 2075, (2002).

10. He, J. H., "Non-Perturbative Methods for Strongly Non-linear Problems," Berlin, dissertation.de Verlag im Internet GmbH, (2006).

11. He, J. H., "Application of Homotopy Perturbation Method to Nonlinear Wave Equations," Chaos, Solitons and Fractals, Vol. 26, No. 3, pp. 695 - 700, (2005).

12. Liao, S. J., "An Approximate Solution Technique Not Depending on Small Parameters: A Special Example," Int. J. Non-Linear Mechanics. Vol. 30, No. 3, pp. 371 - 380, (1995).

13. Liao, S. J., "Boundary Element Method for General Nonlinear Differential Operators," Engineering Analysis with Boundary Elements, Vol. 20, No. 2, pp. 91 - 99, (1997).

14. Razelos, P., and Imre, K., "The Optimum Dimensions of Circular Spines with Variable Thermal Parameters," Journal of Heat Transfer, Vol. 102, pp. 420 - 425, (1980).

15. Unal, H. C., "Determination of the Temperature Distribution in an Extended Surface with a NonUniform Heat Transfer Coefficient," Int. Journal of Heat and Mass Transfer, Vol. 28, pp. 2279 - 2284, (1985).

16. Sen, A. K., and Trinh, S., "An Exact Solution for the Rate of Heat Transfer from a Rectangular spine Governed By A Power-Type Temperature Dependence," ASME Trans. Journal of Heat Transfer, vol. 108 , pp. $457-459,(1985)$.

17. Laor, K., and Kalman, H., "Performanœe and Optimum Dimensions of Different Cooling Spines with a Temperature-Dependent Heat Transfer Coefficient," Int. Journal of Heat and Mass Transfer, Vol. 39, pp. $1993-2003,(1996)$.

Table 1: Parameters for different cross sections of spines

\begin{tabular}{|c|c|c|c|c|}
\hline \hline \multirow{2}{*}{ Parameters } & \multicolumn{4}{|c|}{ Cross Sections } \\
\cline { 2 - 5 } & Rectangular & Circular & Square & Elliptical \\
\hline \hline $\mathcal{L}$ & $\frac{\sqrt{\pi \epsilon_{2}}}{\left(1+\epsilon_{2}\right)} \mathcal{L}_{C}$ & $\mathcal{L}_{C}=d / 4$ & $\frac{\sqrt{\pi}}{2} \mathcal{L}_{C}$ & $\frac{\pi \sqrt{\epsilon_{1}}}{2 E(e)} \mathcal{L}_{C}$ \\
\hline$A_{c}$ & $t b$ & $\pi d^{2} / 4$ & $s^{2}$ & $\pi a b$ \\
\hline$p$ & $2(b+t)$ & $\pi d$ & $4 s$ & $4 a E(e)$ \\
\hline \hline
\end{tabular}




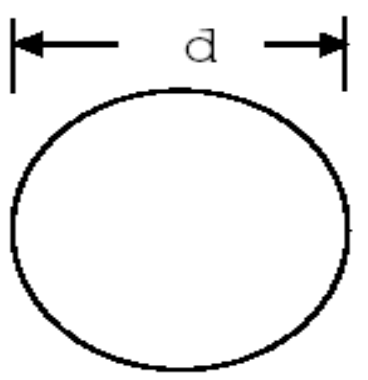

Circular

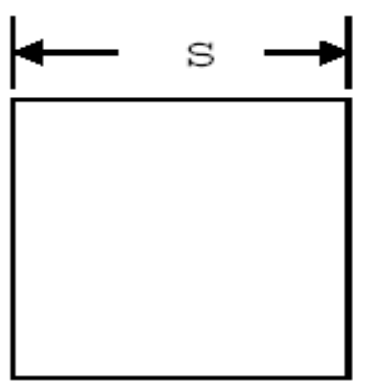

Square

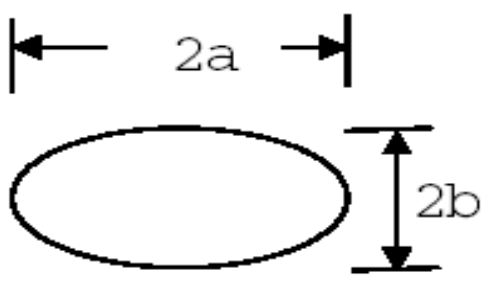

El7iptical

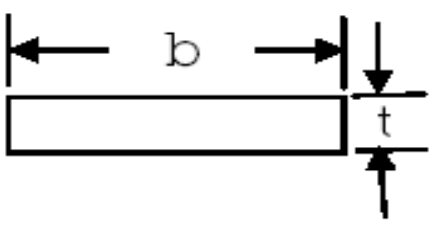

Rectangular

Figure 1: Cross Sections of Spines.
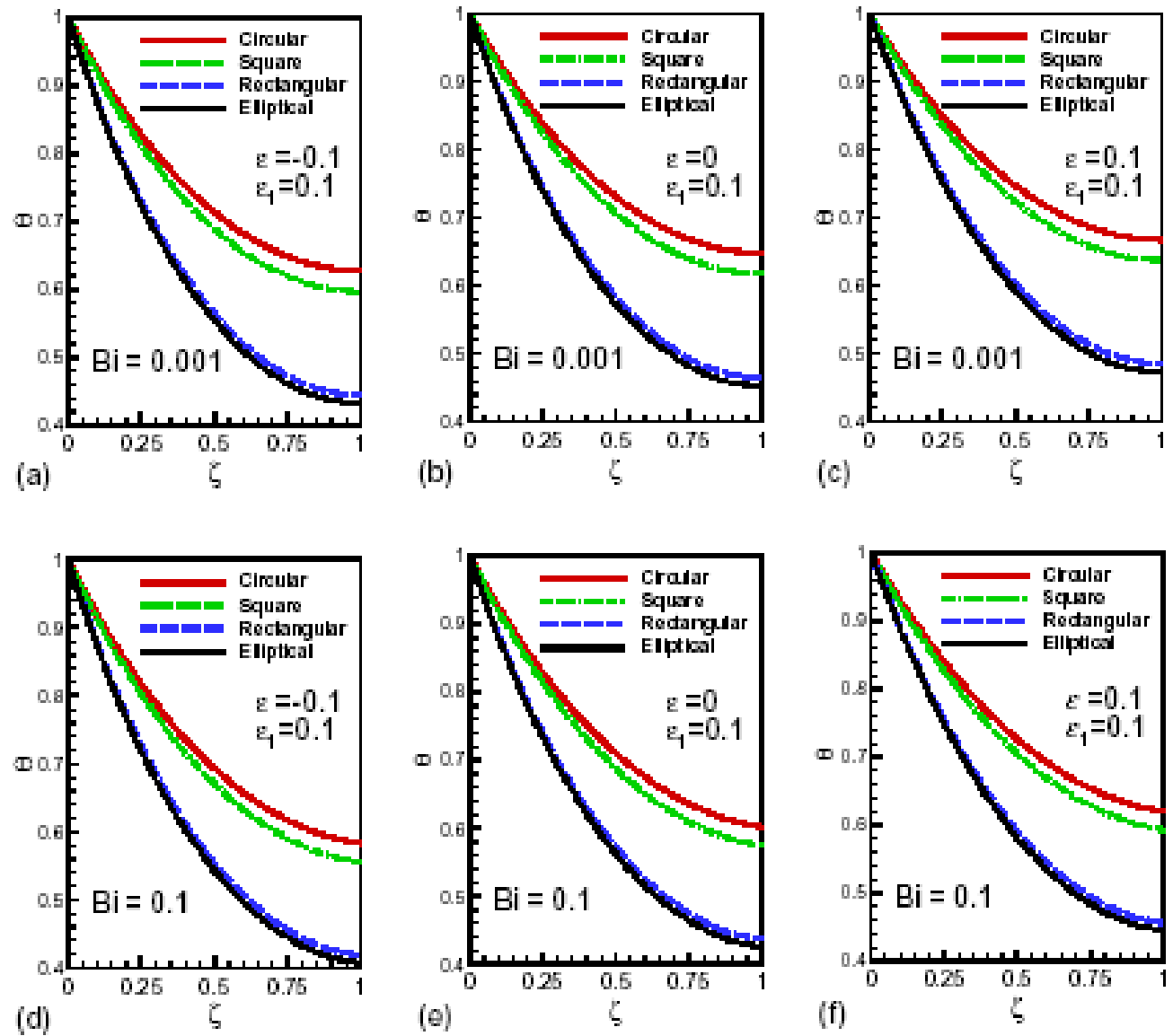

Figure 2: Effect of thermal conductivity and Biot number on temperature distribution along spines. 

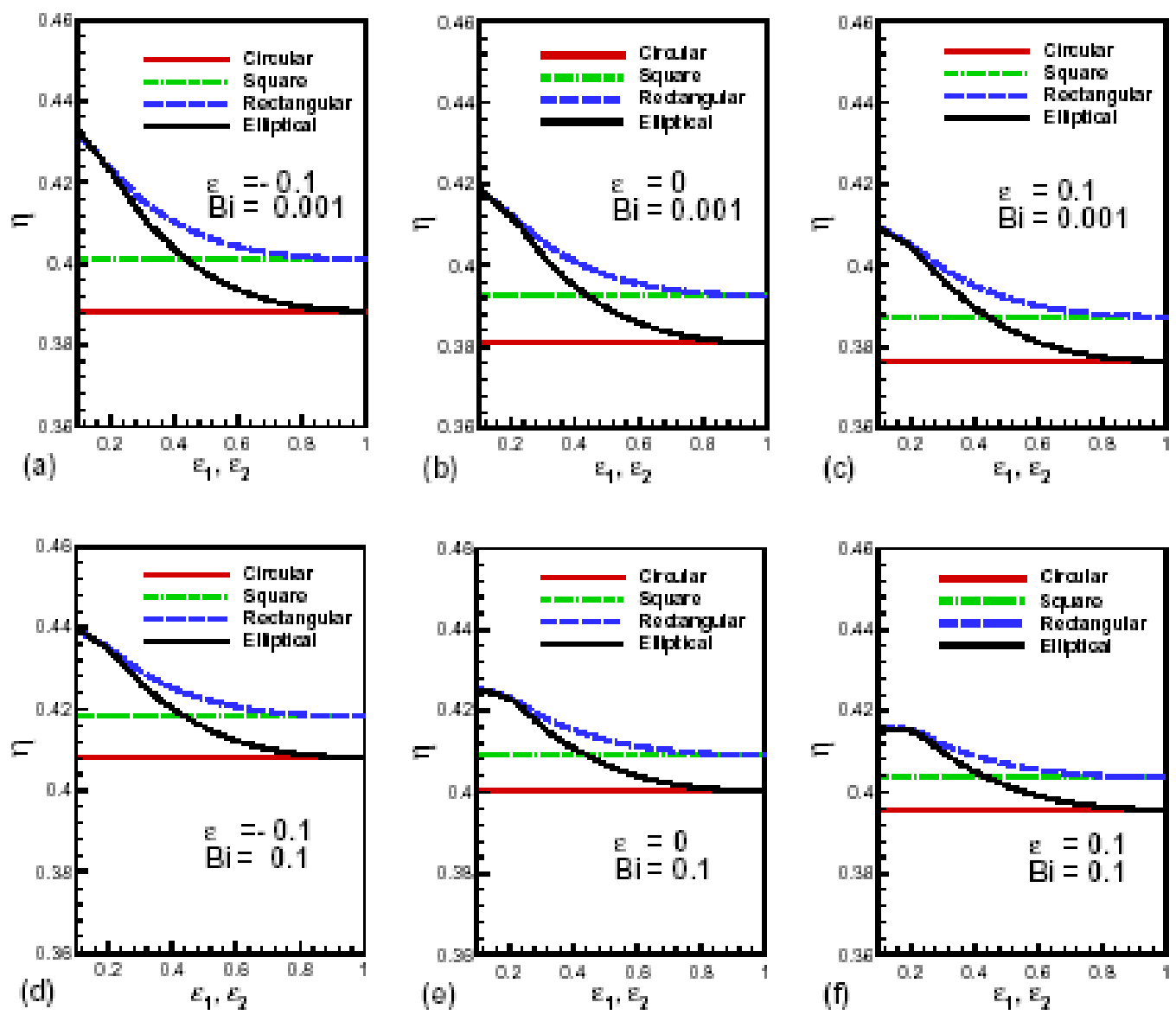

Figure 3: Effect of thermal conductivity and Biot number on efficiency of spines. 